\title{
THE DISCRETENESS OF SPECTRUM FOR HIGHER-ORDER DIFFERENTIAL OPERATORS IN WEIGHTED FUNCTION SPACES
}

\author{
MAOZHU ZHANG ${ }^{凶}$, JIONG SUN and JIJUN AO
}

(Received 18 October 2011; accepted 1 July 2012)

\begin{abstract}
In this paper we consider the discreteness of spectrum for higher-order differential operators in weighted function spaces. Using the method of embedding theorems of weighted Sobolev spaces $H_{p}^{n}$ in weighted spaces $L_{s, r}$, we obtain a new sufficient and necessary condition to ensure that the spectrum is discrete, which can be easily used to judge the discreteness of some differential operators.
\end{abstract}

2010 Mathematics subject classification: primary 34L05; secondary 47E05.

Keywords and phrases: differential operators, spectrum, discreteness.

\section{Introduction}

The spectrum of differential operators is a fundamental and important problem in the theory of differential operators and over the years much attention has been paid to the situation in which the spectrum is discrete. Since Molcanov in 1953 established the celebrated criterion on the discreteness of the spectrum, this result has been developed by many authors [1-7]. Particularly in recent years much work has been done on higher-order differential operators in weighted function spaces, that is, the self-adjoint operators generated by the differential expression

$$
L y=\frac{1}{r^{2}} \sum_{k=0}^{n}(-1)^{k}\left(\rho_{k}^{2} y^{(k)}\right)^{(k)}, \quad x \in \mathbb{R} .
$$

In 1991 and 1994 Edmunds and Sun [2, 7], obtained some conditions to ensure that the spectrum is discrete. In particular, under some assumptions, if $r^{2}$ is bounded below and above by positive constants, then the spectrum of all the self-adjoint extensions is discrete if and only if

$$
\lim _{|x| \rightarrow \infty} \int_{x}^{x+w} \rho_{0}^{2}(t) d t=\infty
$$

The work of the authors is supported by the National Nature Science Foundation of China (grant number 11161030) and Shandong Education Fund of College Scientific Research (grant number J12LI57).

(C) 2012 Australian Mathematical Publishing Association Inc. 0004-9727/2012 \$16.00 
for all $w>0$. Using these results, we can judge the discreteness of some differential operators. However, for some differential operators satisfying similar conditions, these results are hard to deal with. Such examples can be found in Section 3. Motivated by this, we modify the earlier papers [2, 7] and obtain that the spectrum of the operator generated by (1.1) is discrete under the additional assumption that the potential function $\rho_{0}^{2}$ is bounded above and below by positive constants if and only if

$$
\lim _{|x| \rightarrow \infty} \int_{x}^{x+w} r^{2}(t) d t=0
$$

for all $w>0$. This can be easily used to judge the discreteness of the spectrum or the existence of the essential spectrum of a certain class of higher-order differential operators.

As is well known, the theory of the spectrum is related to the embedding of Sobolev space in a Hilbert space. Let $H_{p}^{n}$ and $L_{s, r}$ represent the completion of $C_{0}^{\infty}(R)$ with respect to the norm $\|\cdot\|_{H_{p}^{n}}$ and $\|\cdot\|_{s, r}$ respectively, where

$$
\begin{aligned}
& \|f\|_{H_{p}^{n}}=\left(\sum_{k=0}^{n}\left\|f^{(k)} \rho_{k}\right\|_{p}^{p}\right)^{1 / p}, \\
& \|f\|_{s, r}=\|f r\|_{s} .
\end{aligned}
$$

Our results are based on the following lemma; see [1, 2, 6, 7].

Lemma 1.1. The spectrum of the self-adjoint operator $T$ generated by $L$ is discrete if and only if $H_{2}^{n}$ is compactly embedded in $L_{2, r}$.

This paper is organised as follows. Section 2 is devoted to the main results, including the embedding of $H_{p}^{n}$ in $L_{s, r}$ and the discreteness of the spectrum. In Section 3, some examples are described.

\section{Main results}

Throughout the paper, $n$ will denote a positive integer and $1<p \leq s<\infty, p^{\prime}=$ $p /(p-1) ; I$ will stand for an arbitrary interval in $\mathbb{R}$ of length $|I|$. The coefficients and the weight function satisfy the following fundamental assumptions: $\rho_{0}, \rho_{1}, \ldots, \rho_{n}, r$ are functions on $\mathbb{R}$ which are measurable and positive almost everywhere; it is assumed that $r \in L_{s}^{\mathrm{loc}}(\mathbb{R}), \rho_{0}, \rho_{1}, \ldots, \rho_{n} \in L_{p}^{\mathrm{loc}}(\mathbb{R}), 1 / \rho_{1}, \ldots, 1 / \rho_{n} \in L_{p^{\prime}}^{\mathrm{loc}}(\mathbb{R})$ and $\rho_{0} \notin$ $L_{p}(-\infty, 0) \cup L_{p}(0, \infty)$.

Assume that there exist positive constants $c, c_{1}, c_{2}, c_{3}$ such that:
(A) $\left(\int_{I} \rho_{k}^{-p^{\prime}}\right)^{1 / p^{\prime}}\left(\int_{I} \rho_{k+1}^{p}\right)^{1 / p} \leq c|I|$, for all $\mathrm{I}, k=1,2, \ldots, n-1$;
(B) $\left(\int_{I} \rho_{0}^{-p^{\prime}}\right)^{1 / p^{\prime}}\left(\int_{I} \rho_{1}^{p}\right)^{1 / p} \leq c|I|$, for all I;
(C) $c_{1} \leq \rho_{0}, c_{3} \leq \rho_{n}$ almost everywhere on $\mathbb{R}$;
(D) $\rho_{0} \leq c_{2}$ almost everywhere on $\mathbb{R}$. 
For any $x \in \mathbb{R}$ and a fixed $j \in(0, \infty)$, define

$$
d_{j}(x)=\sup \left\{d>0:\left(\int_{x-\frac{1}{2} d}^{x+\frac{1}{2} d} \rho_{0}^{p} d t\right)^{1 / p} d^{1 / p^{\prime}} \leq j\right\} .
$$

For $k \in(j, \infty)$, define

$$
d_{k}(x)=\sup \left\{d>0:\left(\int_{x-\frac{1}{2} d}^{x+\frac{1}{2} d} \rho_{0}^{p} d t\right)^{1 / p} d^{1 / p^{\prime}} \leq k\right\}
$$

Let

$$
\begin{aligned}
x_{j}^{+}=x+\frac{1}{2} d_{j}(x), \quad x_{j}^{-} & =x-\frac{1}{2} d_{j}(x), \quad \triangle_{j}(x)=\left(x_{j}^{-}, x_{j}^{+}\right), \\
d(x) & =d_{k}(x)-d_{j}(x) .
\end{aligned}
$$

The case of $j=1, k=2$ is a special case here, which can be found in [2, 7]. Here we introduce three new functions $d_{j}(x), d_{k}(x)$ and $d(x)$, by which we can obtain the following theorems.

Theorem 2.1. Suppose that conditions $(A)$ and $(C)$ hold.

(1) If

$$
M=\sup _{x \in \mathbb{R}} M(x)=\sup _{x \in \mathbb{R}} d_{j}(x)^{1 / p^{\prime}}\left(\int_{\Delta_{j}(x)} r^{s}\right)^{1 / s}<\infty,
$$

then $H_{p}^{n}$ is embedded in $L_{s, r}$.

$$
\lim _{|x| \rightarrow \infty} M(x)=0,
$$

then $H_{p}^{n}$ is compactly embedded in $L_{s, r}$.

Proof. This is similar to [2, proof of Theorem 1].

Theorem 2.1 gives conditions sufficient to ensure that the embedding exists or is compact. The following theorem provides a necessary condition for this to happen.

Theorem 2.2. Suppose that conditions (A), (B) and (C) hold.

(1) If $H_{p}^{n}$ is embedded in $L_{s, r}$, then

$$
\sup _{x \in \mathbb{R}}(d(x))^{n} M(x)<\infty .
$$

(2) If $H_{p}^{n}$ is compactly embedded in $L_{s, r}$, then

$$
\lim _{|x| \rightarrow \infty}(d(x))^{n} M(x)=0 .
$$


Proof. Set

$$
f_{x}(t)=(d(x))^{n}\left(d_{j}(x)\right)^{1 / p^{\prime}} g_{x}(t), \quad \forall x \in \mathbb{R}, \forall t \in \mathbb{R},
$$

where

$$
g_{x}(t)= \begin{cases}f\left(2\left(t-x_{k}^{-}\right) / d(x)\right), & x_{k}^{-} \leq t<x_{j}^{-} \\ 1, & x_{j}^{-} \leq t<x_{j}^{+} ; \\ f\left(2\left(x_{k}^{+}-t\right) / d(x)\right), & x_{j}^{+} \leq t<x_{k}^{+} \\ 0, & \mathbb{R} \backslash\left(x_{k}^{-}, x_{k}^{+}\right),\end{cases}
$$

where $f \in C^{\infty}(0,1)$ such that $0 \leq f \leq 1, f(0)=0, f(1)=1, f^{\prime} \in C_{0}^{\infty}(0,1)$, and

$$
\max _{1 \leq i \leq n} \max _{0 \leq t \leq 1}\left|f^{(i)}(t)\right| \leq K \text {. }
$$

Using methods similar to those used to prove [7, Theorem 2], we obtain the results.

So far we have obtained some sufficient conditions or necessary conditions to ensure that the embedding exists or is compact. Below we will use the above conclusions to give a necessary and sufficient condition to ensure that the embedding is compact under the restrictions that the potential function is bounded above and below by two positive constants. Here we extend the functions $d_{1}(x), d_{2}(x)$ and $d(x)$ in [2,7] to $d_{j}(x), d_{k}(x)$ and $d(x)$ in order to obtain the following necessary and sufficient condition to ensure that the spectrum is discrete. We state it in the following theorem.

THEOREM 2.3. Suppose that $\rho_{i}, r$ satisfy the fundamental assumption and conditions (A)-(D). Then $H_{p}^{n}$ is compactly embedded in $L_{s, r}$ if and only if

$$
\lim _{|x| \rightarrow \infty} \int_{x}^{x+w} r^{s} d t=0
$$

for every fixed $w>0$.

Proof. From the condition $\rho_{0} \geq c_{1}$ and Hölder's inequality, together with the definition of $d_{j}(x)$, we can see that

$$
j=d_{j}(x)^{1 / p^{\prime}}\left(\int_{\Delta_{j}(x)} \rho_{0}^{p} d t\right)^{1 / p} \geq c_{1} d_{j}(x),
$$

that is, $d_{j}(x) \leq c_{1}^{-1} j$. By the definition of $d_{j}(x)$ and condition (D), we see that

$$
j=\left(\int_{\Delta_{j}(x)} \rho_{0}^{p} d t\right)^{1 / p} d_{j}(x)^{1 / p^{\prime}} \leq c_{2} d_{j}(x),
$$

that is, $d_{j}(x) \geq c_{2}^{-1} j$. Again by Theorem 2.1(2), the sufficiency of the theorem can be easily obtained. 
If $H_{p}^{n}$ is compactly embedded in $L_{s, r}$, then by Theorem 2.2(2),

$$
\lim _{|x| \rightarrow \infty}(d(x))^{n} d_{j}(x)^{1 / p^{\prime}}\left(\int_{\Delta_{j}(x)} r^{s} d t\right)^{1 / s}=0
$$

for any $k \in(j, \infty)$. Since there exists a positive constant $k>j$ such that $c_{2}^{-1} k-c_{1}^{-1} j>0$, and from $d_{j}(x) \leq c_{1}^{-1} j$, we can get

$$
c_{1}^{-1} k-c_{2}^{-1} j \geq d(x)=d_{k}(x)-d_{j}(x) \geq c_{2}^{-1} k-c_{1}^{-1} j>0 .
$$

Thus we can obtain that

$$
\lim _{|x| \rightarrow \infty} \int_{\Delta_{j}(x)} r^{s} d t=0 .
$$

The required conclusion is attained.

We now consider the spectrum of problem (1.1). The discreteness of spectrum of a self-adjoint operator $T$ generated by differential expressions $L$ is equivalent to the compactness of the embedding of $H_{2}^{n}$ in $L_{2, r}$. Therefore Theorem 2.3 reduces to the following result.

THEOREM 2.4. Suppose that $\rho_{i}, r$ satisfy the fundamental assumption and conditions (A)-(D) with $p=s=2$. Then the spectrum of problem (1.1) is discrete if and only if

$$
\lim _{|x| \rightarrow \infty} \int_{x}^{x+w} r^{2} d t=0
$$

for any fixed $w>0$.

Corollary 2.5. Suppose that there are positive constants $c_{1}, c_{2}$ such that $c_{1} \leq \rho_{i}(x) \leq$ $c_{2}(i=0,1,2, \ldots, n)$ for all $x \in \mathbb{R}$. Then the spectrum of problem (1.1) is discrete if and only if

$$
\lim _{|x| \rightarrow \infty} \int_{x}^{x+w} r^{2} d t=0
$$

for all $w>0$.

Proof. It is easy to prove that the $\rho_{i}$ satisfy all the conditions (A)-(D) with $p=s=2$. Then the result follows from Theorem 2.4.

REMARK 2.6. If some of the $\rho_{i}(i=2,3, \ldots, n-1)$ are zero, these theorems also hold. This is the same as the remark of [2].

\section{Example}

Consider the differential expression

$$
L y=\frac{1}{r^{2}}\left(y^{(4)}-y^{\prime \prime}+\left(1+\frac{3}{4} \sin x\right) y\right), \quad x \in \mathbb{R} .
$$


Here

$$
\rho_{2}^{2}=\rho_{1}^{2}=1, \quad \rho_{0}^{2}=1+\frac{3}{4} \sin x .
$$

If we use [2, Theorem 6] and [7, Theorem 2], we need to compute $d_{1}(x), d_{2}(x)$ and $d(x)$, where

$$
\begin{aligned}
& d_{1}(x)=\sup \left\{d>0:\left(\int_{x-\frac{1}{2} d}^{x+\frac{1}{2} d} \rho_{0}^{p} d t\right)^{1 / p} d^{1 / p^{\prime}} \leq 1\right\}, \\
& d_{2}(x)=\sup \left\{d>0:\left(\int_{x-\frac{1}{2} d}^{x+\frac{1}{2} d} \rho_{0}^{p} d t\right)^{1 / p} d^{1 / p^{\prime}} \leq 2\right\},
\end{aligned}
$$

and

$$
d(x)=d_{2}(x)-d_{1}(x) .
$$

Indeed, we find that these functions are very hard to compute. Thus using the previous results is difficult. However, using the results obtained here we can easily judge the discreteness of the spectrum of the above differential operator. It is easy to see that all the functions $\rho_{0}, \rho_{1}, \rho_{2}$ are bounded below and above. Thus by Corollary 2.5, we have that the spectrum of (3.1) is discrete if and only if

$$
\lim _{|x| \rightarrow \infty} \int_{x}^{x+w} r^{2} d t=0
$$

for every $w>0$.

\section{References}

[1] D. E. Edmunds and W. D. Evans, Spectral Theory and Differential Operators (Oxford University Press, Oxford, 1987).

[2] D. E. Edmunds and J. Sun, 'Embedding theorems and the spectra of certain differential operators', Proc. R. Soc. Lond. A. 434 (1991), 643-656.

[3] I. M. Glazman, 'Direct method of qualitative spectral analysis of singular differential operators', Israel Programme for Scientific Translations, Jerusalem, 1965.

[4] P. E. Müller, Spectral Theory of Ordinary Differential Operators (Ellis Horwood, Chichester, 1981).

[5] P. E. Müller and J. Sun, 'On the discrete spectrum of ordinary differential operators in weighted function spaces', J. Anal. Appl. 14(3) (1995), 637-646.

[6] R. Oinarov and M. Otelbaev, 'A criterion for the discreteness of the spectrum of the general SturmLiouville operator and embedding theorems connected with it', Differ. Uravn. 24 (1988), 584-594.

[7] J. Sun, 'On the spectrum of a class of differential operators and embedding theorems', Acta Math. Sin. (N.S.) 10(4) (1994), 415-427.

\footnotetext{
MAOZHU ZHANG, College of Mathematics and Statistics, Taishan University, Taian 271021, PR China and School of Mathematical Sciences, Inner Mongolia University, Hohhot 010021, PR China e-mail: zhangmaozhu2000@163.com
} 
JIONG SUN, School of Mathematical Sciences, Inner Mongolia University, Hohhot 010021, PR China

e-mail: masun@imu.edu.cn

JIJUN AO, College of Sciences, Inner Mongolia University of Technology, Hohhot 010051, PR China 